\title{
Phase-Code Shift Keyed Probing Signals with Discrete Linear Frequency Modulation and Zero Autocorrelation Zone
}

\author{
Roman N. Ipanov, Member, IEEE
}

\begin{abstract}
Modern synthesized aperture radars (SAR), e.g. space SARs for remote sensing of the Earth, use signals with linear frequency modulation and signals with phase-code shift keying (PCSK) coded by M-sequence (MS) as probing signals. Utilization of PCSK-signals permits an essential improvement of the radar image quality at the stage of its compression on azimuthal coordinate. In this paper, probing signals with zero autocorrelation zone (ZACZ) are synthesized, which signals represent a sequence of two PCSK-pulses with additional linear frequency modulation of sub-pulses in the pulses. A comparative analysis of the correlation characteristics of the synthesized signal and the PCSK-signal coded by MS has been performed. It is shown that in ZACZ, at a mismatch in the Doppler frequency, the level of all side lobes (SL) of the autocorrelation function (ACF) of the synthesized signal is less than the ACF SL level of the PCSK-signal coded by MS. The total ACF of the ensemble of 4 signals has zero SL along the whole time axis $\tau$, and at a mismatch in frequency in ZACZ, it has a lower SL level than the total ACF SLs of the ensemble of 4 PCSK-signals coded by MS.
\end{abstract}

Index Terms-Ambiguity function, autocorrelation function, complementary sequences, pulse train, zero autocorrelation zone.

\section{INTRODUCTION}

$\mathrm{S}$ ynthesized aperture radars (SAR), e.g. space SARs of the Earth remote sensing, due to their operation principle, impose the following requirements on probing signals:

- they must be coherent within the limits of the time interval equal to the aperture synthesis time to provide a high spatial resolution on the azimuth co-ordinate, which is directed along the velocity vector of the space vehicle;

- they must have an intra-pulse modulation to provide a high value of the average emission power and, at the same time, a good spatial resolution on the elevation co-ordinate, which is orthogonal to the space vehicle velocity vector direction. This requirement implies the utilization of the so-called complex signals in SARs, for which signals the product of the effective frequency band and the duration of the probing signals is much higher than 1;

- together with other SAR parameters, they must provide the quality of the radar image, as required in the specifications.

The reported study was funded by RFBR and MCESSM according to the research project № 19-57-44001.

Roman N. Ipanov is with the Moscow Power Engineering Institute (National Research University), Moscow 111250, Russia (e-mail: iproman@ya.ru).
Among various classes of complex signals, only two main classes have yet found practical application in space SARs these are signals with linear frequency modulation (LFM) and signals with phase-code shift keying (PCSK). Space SARs for remote sensing of the Earth have been using LFM-signals up to now $[1-5]$. This situation is determined historically, as LFMsignals were the first complex signals to be used in radar technology, and their properties were studied in detail a long time ago. PCSK-signals were used as probing signals in space SARs mounted on Venus-15 and Venus-16 automatic interplanetary stations designed to provide a radar map of the Venus in 1983-1984 [6], [7]. Truncated M-sequences (MS) were used as the code of those signals.

At the same time, we would like to note a growing interest of radar experts in PCSK-signals [8-11]. This results from the fact that utilization of discrete coding of the coherent ensemble of probing signals in SARs opens the prospect for significant improvement of the radar image quality in terms of parameters related to the properties of total correlation functions of the ensemble. PCSK signals have a number of fundamental advantages:

- the ambiguity function (AF) of the PCSK-signal has a "thumbtack" form, which is close to an ideal one, as opposed to the AF of the LFM-signal, which has a form of a "comb";

- correlation characteristics of the coherent train of reflected probing PCSK-signals can be significantly improved at the stage of signal compression in azimuth when an ensemble of different orthogonal codes is used;

- when the operation of pulse compression by range is performed, no "window" processing is required for the ensemble of PCSK-signals.

Nevertheless, PCSK-signals have shortcomings in comparison with LFM-signals. For instance, a single PCSKsignal for short code sequences has a higher level of the maximum side lobe (SL) of the autocorrelation function (ACF) and a higher integral level of the ACF SL.

In view of the aforesaid, it is expedient to consider PCSKsignals with zero autocorrelation zone (Zero Autocorrelation Zone - ZACZ) [12 - 15] in the area of the central ACF peak as probing signals for SARs. These signals are a periodic sequence (a train) of $M \gg 1$ coherent pulses coded (shift-keyed by phase) by ensembles of complementary or orthogonal sequences.

In space SARs, pauses between the emission of probing signals are used for receiving echoes reflected from the Earth surface, i.e. signal reception and transmission with the same 
antenna are alternated. Therefore, signals with ZACZ considered in [12-15] are not suitable for this purpose, as they have a large number of pulses in the train.

Signals with ZACZ considered in [16] and [17] consist of a minimum number of pulses in the train, i.e. two pulses; however, at mismatch in the Doppler frequency, they have a high enough ACF SL level.

The object of this paper is the synthesis of probing signals with ZACZ consisting of two PCSK-pulses in the train $(M=2)$ and having, at mismatch in the Doppler frequency, an acceptable SL level of the ACF both for single probing signals and the total ACF of the ensemble of PCSK-signals used in the aperture synthesis mode.

One of the varieties of such a signal was considered in [18]. There, the signal is a sequence of two PCSK-pulses with additional linear frequency shift keying of sub-pulses in pulses. In this paper, we consider an additional linear frequency modulation of sub-pulses in pulses.

\section{FORMATION OF THE ZACZ-SIGNAL WITH LINEAR} FREQUENCY MODULATION OF SUB-PULSES IN PULSES

$$
\begin{gathered}
\mathbf{A}_{2, N}=\left(\begin{array}{ll}
\mathbf{A}_{2, N / 2}^{(1)} & \mathbf{A}_{2, N / 2}^{(2)}
\end{array}\right) ; \\
\mathbf{A}_{2, N / 2}^{(1)}=\left(\begin{array}{l}
\mathbf{D}_{1, N / 2}^{i} \\
\mathbf{D}_{1, N / 2}^{j}
\end{array}\right) ; \mathbf{A}_{2, N / 2}^{(2)}=\left(\begin{array}{l}
\mathbf{D}_{1, N / 2}^{i^{\prime}} \\
\mathbf{D}_{1, N / 2}^{j^{\prime}}
\end{array}\right) ; \\
i, j, i^{\prime}, j^{\prime}=1,2, \ldots, N / 2,
\end{gathered}
$$

where $\quad$ sequences $\quad \mathbf{D}_{1, N / 2}^{i}=\left\|d_{i, n}\right\|_{n=1}^{N / 2}, \quad \mathbf{D}_{1, N / 2}^{j}=\left\|d_{j, n}\right\|_{n=1}^{N / 2} \quad$ and $\mathbf{D}_{1, N / 2}^{i^{\prime}}=\left\|d_{i^{\prime}, n}\right\|_{n=1}^{N / 2}, \quad \mathbf{D}_{1, N / 2}^{j^{\prime}}=\left\|d_{j^{\prime}, n}\right\|_{n=1}^{N / 2} \quad$ are complementary sequences (CS); $i, j$ and $i^{\prime}, j^{\prime}$ are numbers of paired CSs; $i^{\prime}$ is the number of the CS, which is adjacent to the CS with the number $i$, and $j^{\prime}$ is the number of the CS, which is adjacent to the CS with the number $j$ [19].

The PCSK-signal (2) coded by rows of the matrix $\mathbf{A}_{2, N}$ from (3) is called a coherent complementary signal (CCS). We call this signal "coherent" because the coherence of PCSK pulses must be maintained in the train. In addition, we call it "complementary" because the pulses are encoded by
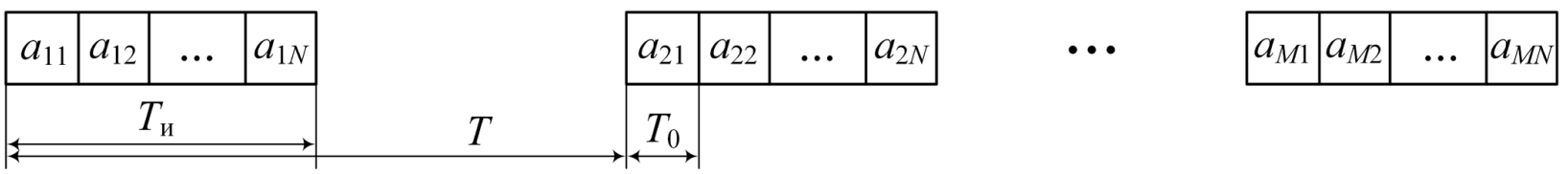

Fig. 1. An envelope of a train of $M$ PCSK-pulses

Let us consider a radar signal representing a sequence (a train) of $M$ pulses (Fig. 1) shift-keyed in phase by an ensemble of $M$ sequences [18]. Each pulse with the duration $T_{\mathrm{p}}$ consists of $N$ sub-pulses (discretes) with the duration of $T_{0}=T_{\mathrm{p}} / N$ each. The pulse repetition period is $T=q T_{\mathrm{p}}=q N T_{0}$, where $q \geq 2$ is the off-duty factor of the pulses. The ensemble of $M$ sequences can be defined by the following matrix:

$$
\mathbf{A}_{M, N}=\left\|a_{i, n}\right\|_{i, n=1}^{M, N}, a_{i, n}=\exp \left(\mathrm{j} \pi \tilde{a}_{i, n}\right),
$$

where $\tilde{\mathbf{A}}_{M, N}=\left\|\tilde{a}_{i, n}\right\|_{i, n=1}^{M, N}$ is the matrix of the binary code.

A complex envelope of the signal under investigation will have the following form:

$$
\dot{u}(t)=\sum_{i=1}^{M} \sum_{n=1}^{N} a_{i, n} S_{n}\left(t-(n-1) T_{0}-(i-1) T\right),
$$

where $S_{n}\left(t-(n-1) T_{0}\right),(n-1) T_{0} \leq t<n T_{0}$, is the envelope of the $n$-th discrete of the pulse.

At $M=2$, the coding matrix (1) can be written as follows [18]: complementary sequences.

Let us consider CCSs with additional frequency modulation of the discretes of train pulses under the linear law (CCS-LFM), as shown in Fig. 2.

The frequency variation law within the limits of the $n$-th discrete is as follows (taking into account Fig. 2):

$f_{n}(t)=\left\{\begin{array}{l}\frac{F_{\mathrm{S}}}{T_{0}} t, \text { for } n=1, \ldots, N / 2 ; \\ F_{\mathrm{S}}-\frac{F_{\mathrm{S}}}{T_{0}} t, \text { for } n=N / 2+1, \ldots, N,\end{array}\right.$ at $0 \leq t<T_{0}$,

where $n=1,2, \ldots, N=2^{k+1}$ is the number of the discrete in the CCS pulse; $F_{\mathrm{S}}=b / T_{0}$ is the width of the CCS-LFM spectrum.

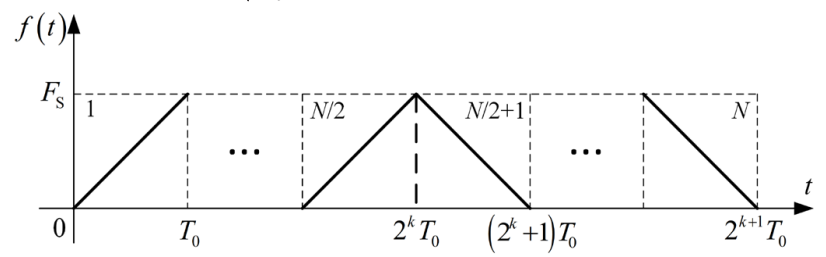

Fig. 2. LFM law of discretes within the limits of the CCS pulse

Then, the phase variation law within the limits of the $n$-th discrete is as follows: 


$$
\varphi_{n}(t)=\left\{\begin{array}{l}
\pi b\left(\frac{t}{T_{0}}\right)^{2}, \text { for } n=1, \ldots, N / 2 \\
2 \pi b \frac{t}{T_{0}}-\pi b\left(\frac{t}{T_{0}}\right)^{2}, \text { for } n=N / 2+1, \ldots, N .
\end{array}\right.
$$

Thus, the complex envelope (2) of the CCS-LFM is as follows:

$$
\begin{aligned}
& \dot{u}(t)= \\
& =\left\{\begin{array}{l}
\sum_{n=1}^{N} S_{0}\left(t-(n-1) T_{0}\right) \exp \left\{\mathrm{j} \pi\left(b\left(\frac{t}{T_{0}}\right)^{2}+\tilde{a}_{1, n}\right)\right\}+ \\
+\sum_{n=1}^{N} S_{0}\left(t-(n-1) T_{0}-T\right) \exp \left\{\mathrm{j} \pi\left(b\left(\frac{t}{T_{0}}\right)^{2}+\tilde{a}_{2, n}\right)\right\}, \\
\text { for } n=1, \ldots, N / 2 ; \\
\sum_{n=1}^{N} S_{1}\left(t-(n-1) T_{0}\right) \exp \left\{\mathrm{j} \pi\left(2 b \frac{t}{T_{0}}-b\left(\frac{t}{T_{0}}\right)^{2}+\tilde{a}_{1, n}\right)\right\}+ \\
+\sum_{n=1}^{N} S_{1}\left(t-(n-1) T_{0}-T\right) \exp \left\{\mathrm{j} \pi\left(2 b \frac{t}{T_{0}}-b\left(\frac{t}{T_{0}}\right)^{2}+\tilde{a}_{2, n}\right)\right\}, \\
\text { for } n=N / 2+1, \ldots, N .
\end{array}\right.
\end{aligned}
$$

where $\left\|\tilde{a}_{i, n}\right\|_{i, n=1}^{2, N}$ is the matrix composed from the adjacent pairs of the binary D-code $[17,19], \quad a_{i, n}=\exp \left(\mathrm{j} \pi \tilde{a}_{i, n}\right)$, $\mathbf{A}_{2, N}=\left\|a_{i, n}\right\|_{i, n=1}^{2, N}$ from (3).

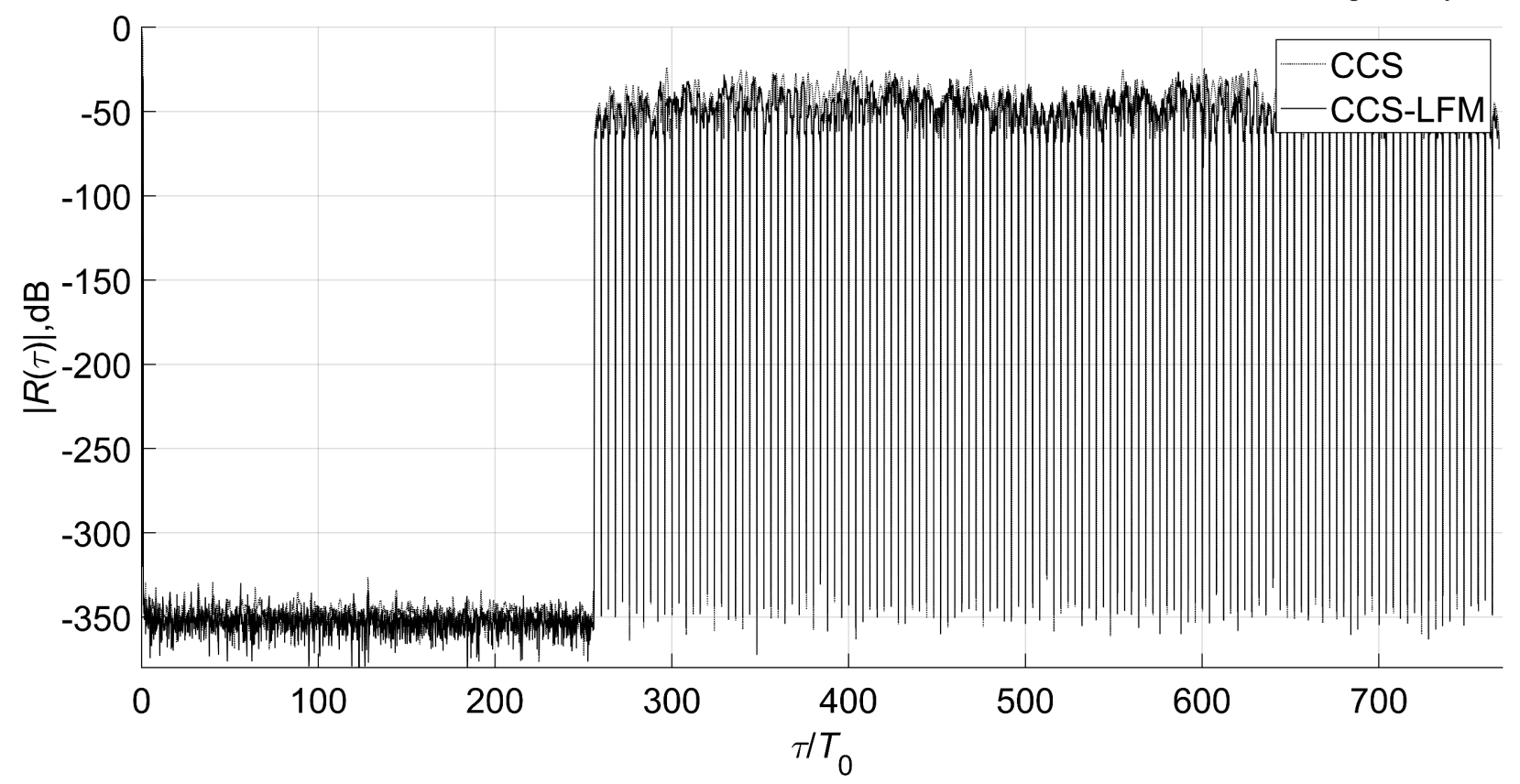

Fig. 3. ACF of CCS (dotted line) and CCS-LFM (solid line) 
It follows from Table 1 that in case of a mismatch by frequency, the maximum ACF SL level of CCS-LFM in ZACZ is lower than the maximum ACF SL level of CCS more than by $6.5 \mathrm{~dB}$, and the rms SL level - more than by $6 \mathrm{~dB}$. Besides, outside ZACZ, the rms ACF SL level of CCS-LFM is lower than the respective ACF SL level of CCS more than by $6 \mathrm{~dB}$.

From [14], [20], we know that pseudo-noise signals are the optimum discrete signals, i.e., signals with a minimum level of the maximum ACF SL. The most known and widely used example of such signals is a truncated $\mathrm{M}$-sequence, in which the minimax SL level of the normalized ACF aspires to $1 / \sqrt{N_{\mathrm{M}}}$ with $N_{\mathrm{M}}$ growth, where $N_{\mathrm{M}}$ is the period of the M-sequence.

TABLE 1

VALUES OF CORRELATION CHARACTERISTICS OF CCS, CCS-LFM AND MS

\begin{tabular}{|c|c|c|c|c|}
\hline \multicolumn{2}{|r|}{$F$} & 0 & $0.3 \Delta F$ & $0.5 \Delta F$ \\
\hline \multirow{3}{*}{$\begin{array}{c}R_{\max }, \\
\mathrm{dB}\end{array}$} & $\mathrm{CCS}$ & -23.8 & -23.8 & -23.8 \\
\hline & CCS-LFM & -26.3 & -26.3 & -26.4 \\
\hline & $\mathrm{MS}$ & -27.3 & -27.8 & -25.2 \\
\hline \multirow{3}{*}{$\begin{array}{c}R_{\mathrm{rms}} \\
\mathrm{dB}\end{array}$} & CCS & -34.9 & -34.8 & -34.7 \\
\hline & CCS-LFM & -41.2 & -41.1 & -41.0 \\
\hline & $\mathrm{MS}$ & -35.2 & -33.8 & -32.4 \\
\hline \multirow{2}{*}{$\begin{array}{c}R_{\mathrm{z} \max }, \\
\mathrm{dB} \\
\end{array}$} & $\mathrm{CCS}$ & -330 & -24.7 & -20.8 \\
\hline & CCS-LFM & -329 & -31.5 & -27.7 \\
\hline \multirow{2}{*}{$\begin{array}{c}R_{\mathrm{z} \mathrm{rms}}, \\
\mathrm{dB}\end{array}$} & $\mathrm{CCS}$ & -343 & -38.6 & -34.7 \\
\hline & CCS-LFM & -349 & -44.9 & -41.0 \\
\hline
\end{tabular}

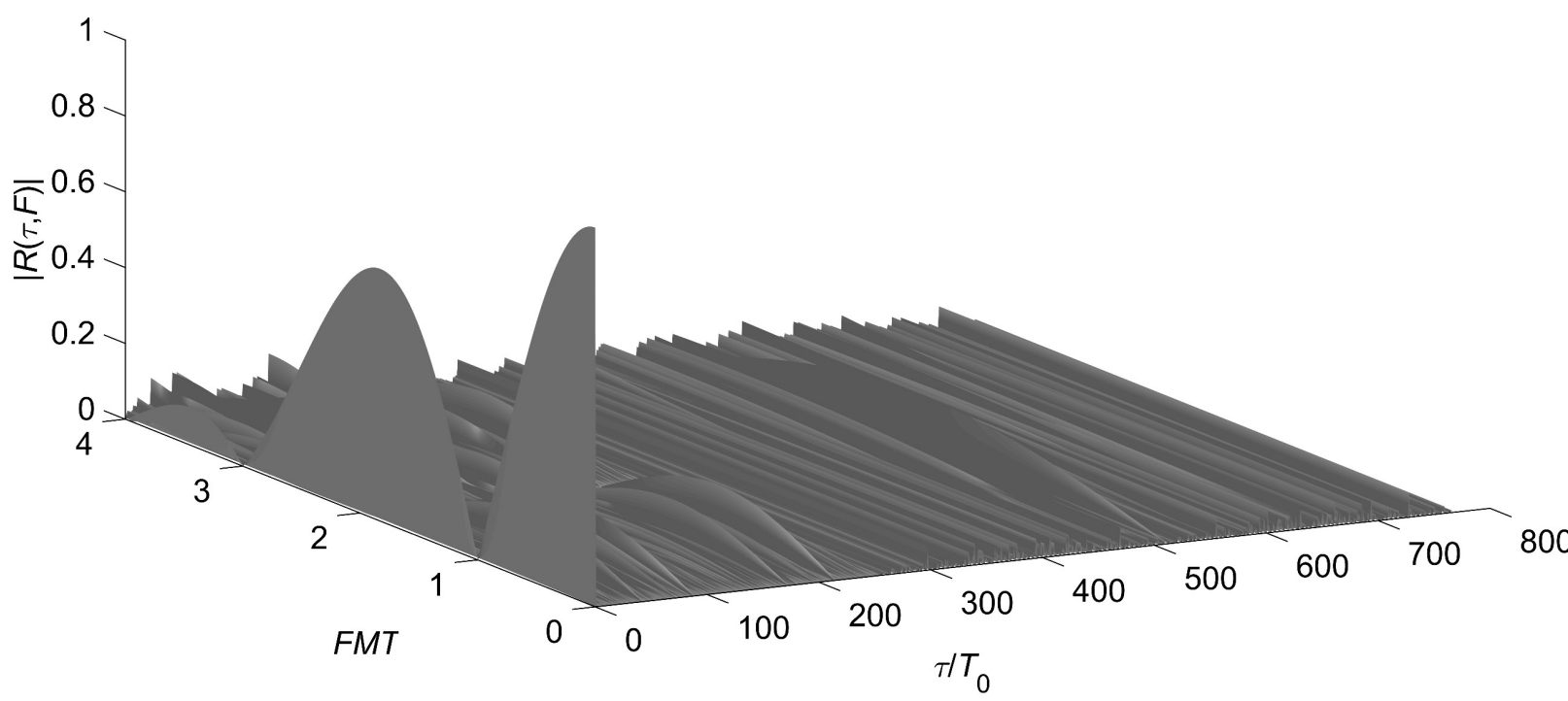

Fig. 4. The ambiguity function of CCS
Table 1 provides values of the correlation characteristics of the considered MS for three sections of the AF with planes $F=0, F=0.3 \Delta F$ and $F=0.5 \Delta F$, and Fig. 6 shows ZACZ for the examined CCS and CCS-LFM signals and a part of the MS ACF at a mismatch in frequency $F=0.3 \Delta F$.

It follows from Fig. 6 and Table 1 that in case of a mismatch by frequency $F=0.3 \Delta F$ and $F=0.5 \Delta F$, the maximum and rms SLs levels of the ACF of the CCS-LFM in ZACZ are less than the corresponding SLs levels of the ACF of the MS. Modeling results showed the validity of this result at $|F|<\Delta F$.

\section{COMPARATIVE ANALYSIS OF TOTAL ACFs OF THE CCS- LFM ENSEMBLE AND CYCLIC MS ENSEMBLE}

As we mentioned earlier, in space SARs, PCSK signals modulated in phase by truncated MSs were used as probing signals. However, in adjacent sensing cycles, for maximum ACF SL suppression depending on the necessary time for the aperture synthesis and other SAR parameters, MSs with different generating polynomials and/or different cyclic shifts were used [6].

The maximum SL suppression of the total ACF of the MS ensemble is achieved by utilization of the complete cyclic MS ensemble with the number of discretes in the pulse equal to its period, i.e., at $N=N_{\mathrm{M}}$. In addition, the adjacent MSs are shifted to each other for one element, and the number of MSs in the ensemble is equal to $N$. Then, for the total ACF of the complete cyclic ensemble of $N \mathrm{MSs}$ with the length of the one period, the
Let us perform a comparative analysis of the correlation characteristics of considered CCSs with the PCSK-signal modulated in phase by an M-sequence (further simply MS) with the period $N_{\mathrm{M}}=511$. To align the levels of the ACF main lobes of CCS and MS, we will add one element from the adjacent period to its period, i.e., the number of discretes in the MS pulse will be $N=N_{\mathrm{M}}+1=512$. following equation may be formulated:

$r_{m}^{+}=\sum_{i=1}^{N} r_{m}^{i} ;|m|=0,1, \ldots, N-1$,

where $r_{m}^{i}$ is non-normalized ACF of $i$-th MS. 
After formulating equations for ACFs of each of the $N$ MSs and summing them up according (7), we will find out that

$r_{m}^{+}=(N-|m|)\left(r_{m}+r_{N-m}\right)$,

where $r_{m}=\sum_{n=1}^{N-m} a_{n} a_{n+m}$ is the aperiodic ACF of the current part of the MS with the length of one period; $r_{N-m}=\sum_{n=1}^{m} a_{n} a_{n+N-m}$ is the aperiodic ACF of the adjacent (from the right) part of the MS.

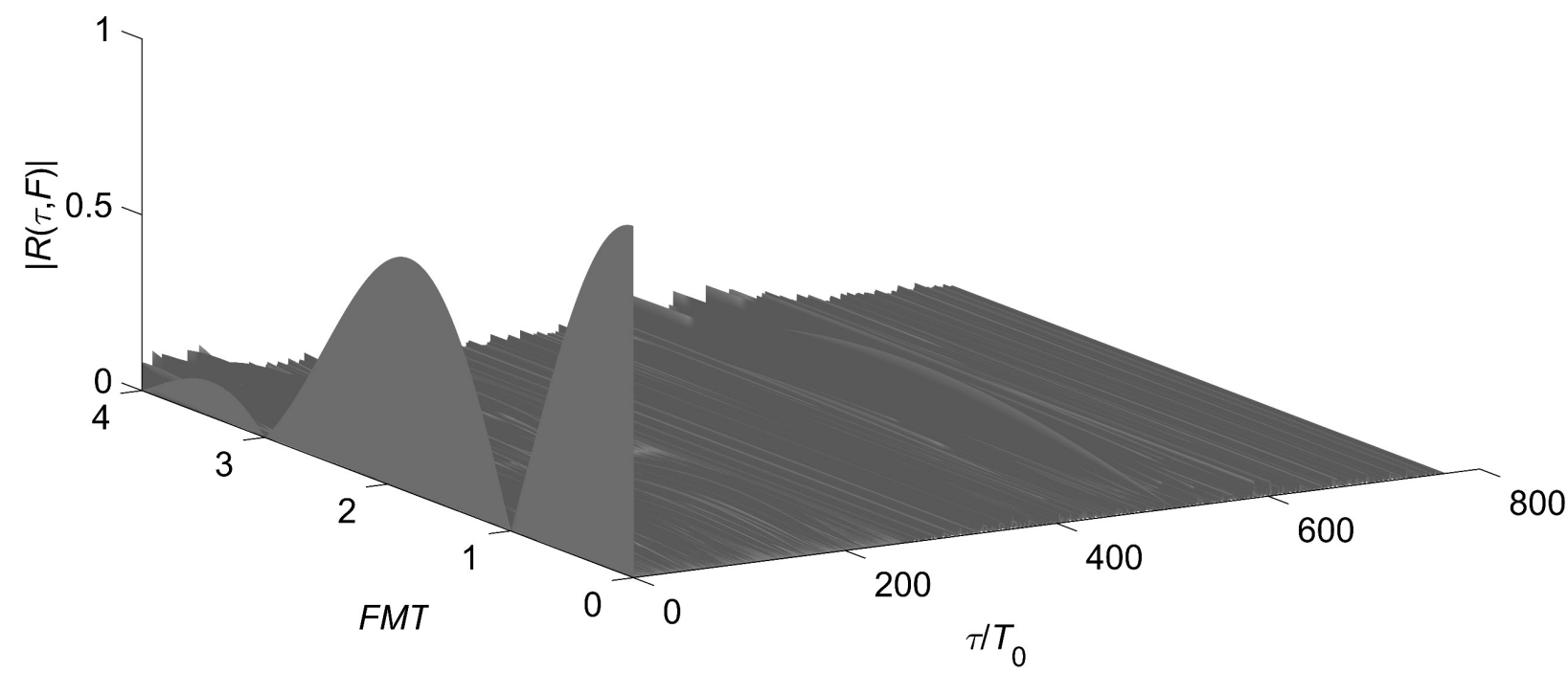

Fig. 5. The ambiguity function of CCS-LFM

It is known from [20] that the sum in (8) determines the periodic $\mathrm{ACF}$ of the $\mathrm{MS}$ with a period $N=N_{\mathrm{M}}$, i.e., $r_{m}+r_{N-m}=r_{m}^{\mathrm{p}}$, where $r_{m}^{\mathrm{p}}=\sum_{n=1}^{N} a_{n} a_{n+m}$ at $|m|=0,1, \ldots, N-1$. It is also known that for MS $r_{m}^{\mathrm{p}}=-1$ at $|m|=1,2, \ldots, N-1$.

Thus, from (8) we obtain that

$r_{m}^{+}=(N-|m|) r_{m}^{\mathrm{p}}=-N+|m|$ at $|m|=1,2, \ldots, N-1$,

from which the normalized total ACF of the complete cyclic ensemble of MS is:

$R_{m}^{+}=-\frac{1}{N}+\frac{|m|}{N^{2}}$ at $|m|=1,2, \ldots, N-1$

The SL level of the total ACF of the complete cyclic MS ensemble can be considered as the minimum possible limit. The lesser MSs the cyclic ensemble contains, the higher SL level of the total ACF is. We shall consider an ensemble consisting of four MSs shifted to each other for 128 elements to be the cyclic ensemble with the maximum SL level of the total ACF.

Table 2 provides the values of the total ACFs of the complete cyclic ensemble of $511 \mathrm{MSs}$ and the cyclic ensemble of $4 \mathrm{MSs}$ for three AF sections with planes $F=0, F=0.3 \Delta F$ and $F=0.5 \Delta F$. In this table, $R_{\Sigma \max }$ and $R_{\Sigma \text { rms }}$ are the relative maximum SL level of the total ACF and the relative rms SL level of the total ACF, respectively. From Table 2 we can see that the maximum SL level of the total ACF of the complete cyclic ensemble is lower than the maximum SL level of the cyclic ensemble of four MSs more than by $15.5 \mathrm{~dB}$, and for rms SL level - more than by $12 \mathrm{~dB}$.

Further, we will look at the values of the total ACF of the CCS-LFM ensemble. According to (3), there is a total of
$N / 2=2^{k}$ CCSs formed from the adjacent AS pairs of the $k$ order and forming the complete CCS ensemble with the number of discretes in the pulse $N=2^{k+1}$.

TABLE 2

VALUES OF THE TOTAL ACF OF ENSEMBLES OF 511 MSs, 4 MSs AND 4 CCS-LFM

\begin{tabular}{|c|c|c|c|c|}
\hline \multicolumn{2}{|c|}{$F$} & 0 & $0.3 \Delta F$ & $0.5 \Delta F$ \\
\hline \multirow{3}{*}{$R_{\Sigma \max }, \mathrm{dB}$} & $4 \mathrm{MS}$ & -38.6 & -39.9 & -39.8 \\
\hline & $511 \mathrm{MS}$ & -54.2 & -55.5 & -58.1 \\
\hline & \multirow{2}{*}{$\begin{array}{l}\text { CCS- } \\
\text { LFM }\end{array}$} & -324 & -30.7 & -26.4 \\
\hline$R_{\Sigma z \max }, \mathrm{dB}$ & & -327 & -43.5 & -35.4 \\
\hline \multirow{3}{*}{$R_{\Sigma \mathrm{rms}}, \mathrm{dB}$} & $4 \mathrm{MS}$ & -46.7 & -46.7 & -46.6 \\
\hline & $511 \mathrm{MS}$ & -58.9 & -59.7 & -61.1 \\
\hline & \multirow{2}{*}{$\begin{array}{l}\text { CCS- } \\
\text { LFM }\end{array}$} & -348 & -57.3 & -52.9 \\
\hline$R_{\Sigma \mathrm{z} \mathrm{rms}}, \mathrm{dB}$ & & -345 & -67.0 & -59.3 \\
\hline
\end{tabular}




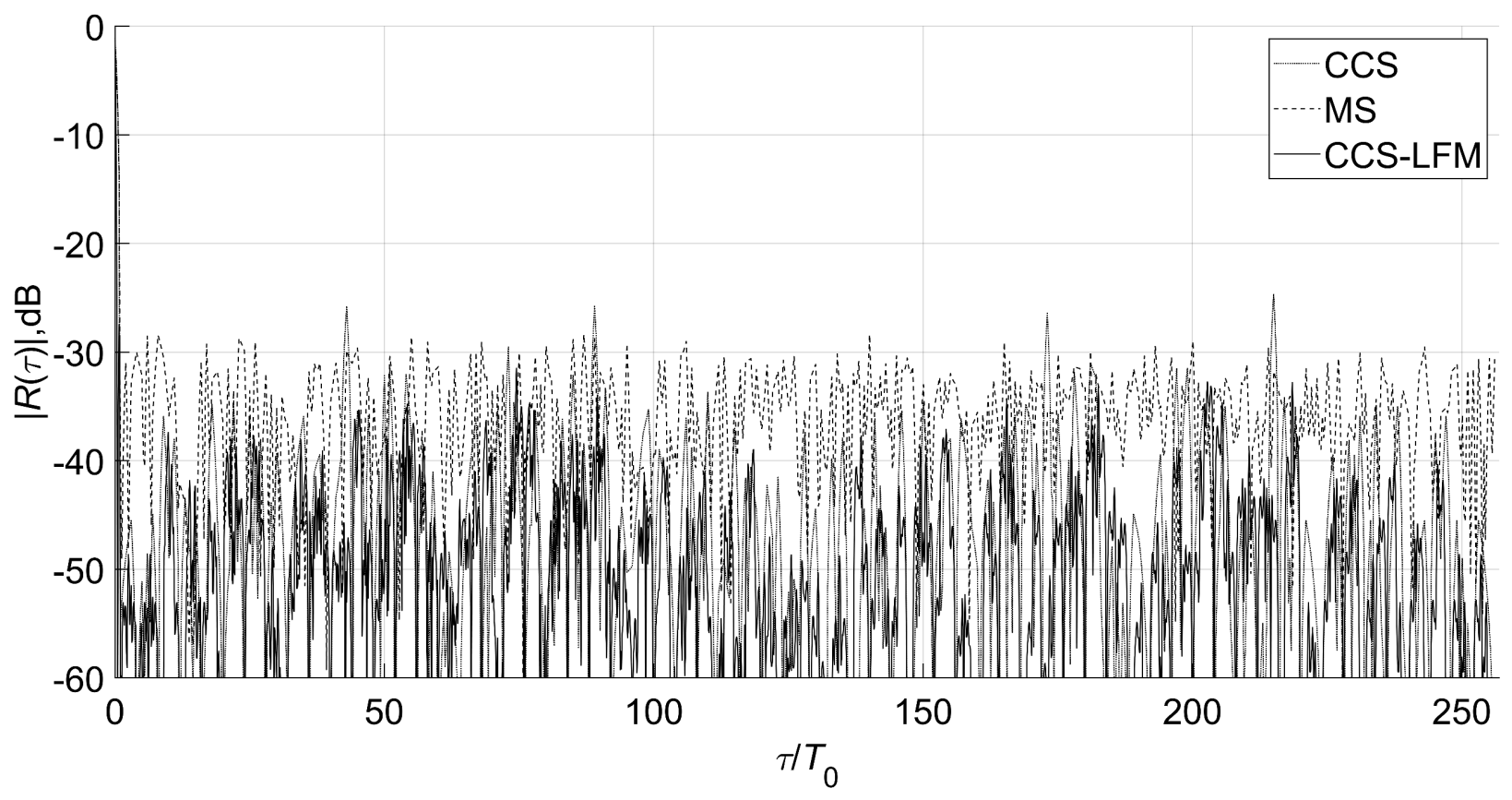

Fig. 6. ZACZ of CCS, CCS-LFM and a part of the MS ACF at $F=0.3 \Delta F$

Modeling results show that the total ACF of the ensemble of four CCSs (with and without modulation of the discrete) with adjacent numbers in the complete ensemble has zero SLs along the whole time axis $\tau$ of the ACF. In this case, the number of the ensemble $j$ of four CCSs with the number of discretes in the pulse being $N=2^{k+1}$ is connected with the CCS number $i$ in the complete ensemble of $N / 2$ using the following equation:

$$
j=\left\lfloor\frac{i-1}{4}\right\rfloor+1 ; i=1,2, \ldots, 2^{k} ; j=1,2, \ldots, 2^{k-2},
$$

where $\lfloor z\rfloor$ is the integer part of the number $z$.

Fig. 7 and 8 show the total ACF $R_{\Sigma}(\tau)$ and the total AF $R_{\Sigma}(\tau, F)$ of $j=1$ ensemble of four CCS-LFM with the number of discretes in the pulse $N=256$ and the base $b=4$, respectively. The off-duty factor is $q=2$.

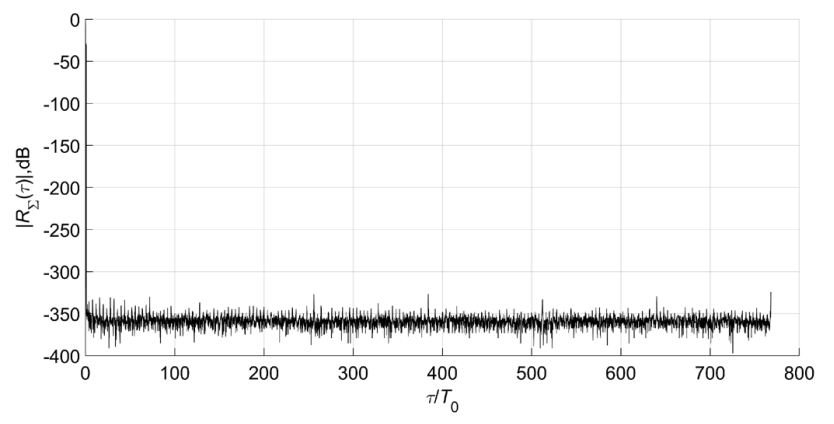

Fig. 7. Total ACF of the ensemble of 4 CCS-LFM

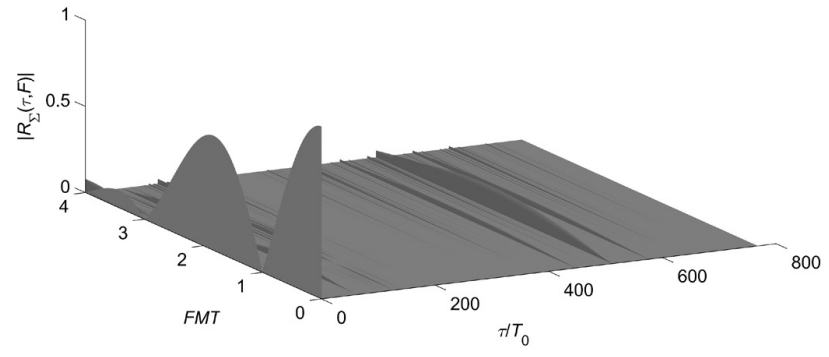

Fig. 8. Total AF of the ensemble of four CCS-LFM

Table 2 also provides the values of the total ACF of the ensemble of four CCS-LFM under consideration for three AF sections with planes $F=0, F=0.3 \Delta F$ and $F=0.5 \Delta F$, and Fig. 9 shows the total ACF of the ensemble of four CCS-LFM, cyclic ensemble of four MSs and complete cyclic ensemble of $511 \mathrm{MSs}$ at mismatch in frequency $F=0.3 \Delta F$. In the Table 2, $R_{\Sigma \text { max }}$ and $R_{\Sigma z \max }$ are relative maximum SL levels of the total $\mathrm{ACF}$ outside and inside of ZACZ, respectively; $R_{\Sigma \text { rms }}$ and $R_{\Sigma \mathrm{z} \text { rms }}$ are relative rms SL levels of the total ACF outside and inside of ZACZ, respectively.

It follows from Fig. 9 and Table 2 that in case of a mismatch by frequency $F=0.3 \Delta F$, the maximum and rms SLs levels of the total ACF of the ensemble of four CCS-LFM are less than the corresponding SLs levels of the total ACF of the ensemble of four MS. Modeling results showed the validity of this result at $|F|<0.35 \Delta F$. 


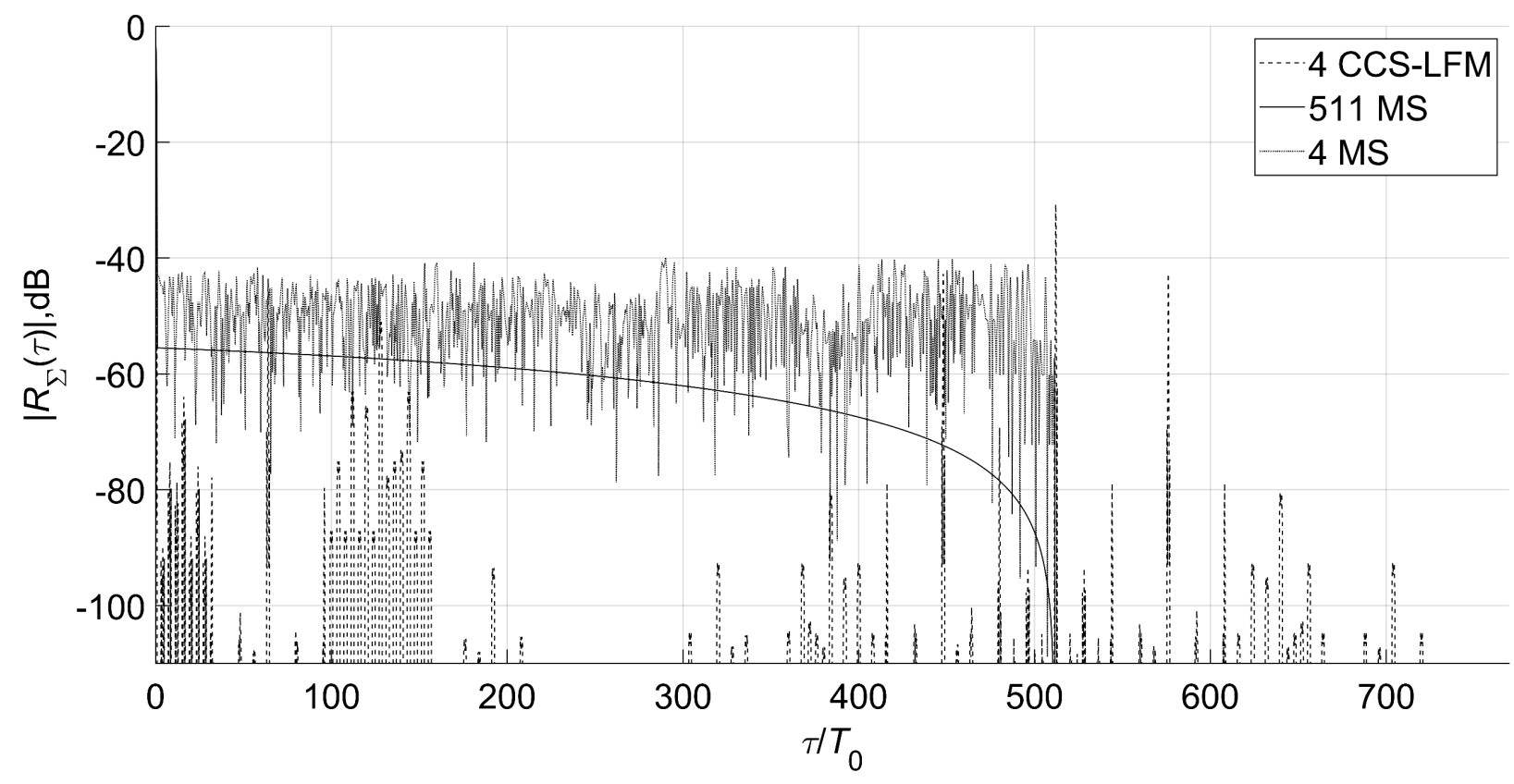

Fig. 9. Total ACFs of the ensembles of four CCS-LFM (dashed line), four MSs (dotted line) and 511 MSs (solid line) at $F=0.3 \Delta F$

\section{CONCLUSION}

This paper addresses probing PCSK-signals (train type) having a zero autocorrelation zone (ZACZ) and called coherent complementary signals (CCS). We prove that such signals have $\mathrm{ZACZ}$ and determine requirements to the coding matrix. Since ZACZ exists for CCS at zero mismatch in the Doppler frequency only, and a mismatch in frequency causes rather a high side lobe level in ZACZ, CCS with additional frequency modulation of closed sub-pulses (discretes) of the train pulses under linear law (CCS-LFM) have been considered for possible suppression of the SL. A comparative analysis of the correlation characteristics of CCS-LFM and CCSs without modulation of discretes at mismatch in the Doppler frequency has been performed. For this, we have assumed the same quantity of discretes in the train pulses. Our analysis has shown that in case of a mismatch by frequency in ZACZ, the maximum ACF SL level of CCS-LFM is lower than the maximum ACF SL level of CCS more than by $6.5 \mathrm{~dB}$, and the rms SL level - more than by $6 \mathrm{~dB}$. We have also performed a comparative analysis of the correlation characteristics of CCS-LFM and CCSs without discrete modulation with PCSK-signal shift-keyed in phase by M-sequence, which has the same number of discretes in the pulse with the number of discretes in two pulses of the CCS train. The analysis has shown that in case of a mismatch by frequency $F=0.3 \Delta F$ and $F=0.5 \Delta F$, the maximum and rms SLs levels of the ACF of the CCS-LFM in ZACZ are less than the corresponding SLs levels of the ACF of the MS. Modeling results showed the validity of this result at $|F|<\Delta F$. We have also performed a comparative analysis of the total ACFs of the CCS-LFM ensemble and the cyclic M-sequence ensemble at mismatch in the Doppler frequency. This analysis has shown that the total ACF of the ensemble of four CCSs (with and without discrete modulation) with adjacent numbers in the complete ensemble has zero SLs along the whole time axis $\tau$ of the ACF. The analysis has shown also that at mismatch in frequency in ZACZ, the maximum and rms SLs levels of the total ACF of the ensemble of four CCS-LFM are less than the corresponding SLs levels of the total ACF of the ensemble of four MS. Modeling results showed the validity of this result at $|F|<0.35 \Delta F$.

\section{REFERENCES}

[1] D. R. Wehner, High Resolution Radar, 2nd ed. Norwood, MA, USA: Artech House, 1994.

[2] N. Ganveer, G. Vishal, R. S. Rao and V. Biradar, "SAR implementation using LFM signal," in Proc. 2016 IEEE Int. Conf. on Recent Trends in Electron. Inform. \& Commun. Techn., Bangalore, India, 2016, pp. 1094-1097, DoI: 10.1109/RTEICT.2016.7808000.

[3] F. Liu, S. Mu, W. Lyu, W. Li and T. Ge, "MIMO SAR Waveform Separation Based on Costas-LFM Signal and Co-arrays for Maritime Surveillance," Chinese J. of Electron., vol. 26, no. 1, pp. 211-217, Jan. 2017, Dor: 10.1049/cje.2016.11.015.

[4] N. Pallavi, P. Anjaneyulu, P. B. Reddy, V. Mahendra and R Karthik, "Design and implementation of linear frequency modulated waveform using DDS and FPGA," in Proc. 2017 Int. Conf. Electron. Commun. and Aeros. Technol., Coimbatore, India, 2017, pp. 237-241, Dor: 10.1109/ICECA.2017.8212806.

[5] Q. Wu, X. Liu, J. Liu, F. Zhao and S. Xiao, "A Radar Imaging Method Using Nonperiodic Interrupted Sampling Linear Frequency Modulation Signal," IEEE Sensors J., vol. 18, no. 20, pp. 8294-8302, Aug. 2018, DoI: 10.1109/JSEN.2018.2865531.

[6] Yu. N. Aleksandrov, A. T. Bazilevskii, V. A. Kotel'nikov, G. M. Petrov, O. N. Rzhiga and A. I. Sidorenko, "A planet rediscovered: results of Venus radar imaging from the Venera 15 and Venera 16 spacecraft," Sov. Sci. Rev., E, Astrophys. Space Phys., vol. 6, no. 1, pp. 61-101, Aug. 1988. 
[7] V. L. Barsukov et al., "The geology and geomorphology of the Venus surface as revealed by the radar images obtained by Veneras 15 and 16," in Proc. 16th Lunar and Planet. Sci. Conf., vol. 91, no. B4, 1986, pp. D378-D398, DoI: 10.1029/JB091iB04p0D378.

[8] K. Jumani and K. Sarabandi, "An investigation of PN sequences for multistatic SAR/InSAR applications," in Proc. 2007 IEEE Int. Geoscience and Remote Sensing Symp., Barcelona, Spain, 2007, pp. 132-137, DOI: 10.1109/IGARSS.2007.4422994.

[9] N. Salerno, L. Simone, S. Cocchi, V. Piloni, M. Maffei and O. Cocciolillo, "Wideband arbitrary waveform generator for enhanced spaceborne SAR," in Proc. 2008 Europe Radar Conf., Amsterdam, Netherlands, 2008, pp. 416-419.

[10] S. F. Li, J. Chen, L. Q. Zhang and Y.Q. Zhou, "Image formation algorithm for missile borne MMW SAR with phase coded waveform," in Proc. 2009 IET Int. Radar Conf., Guilin, China, 2009, pp. 1-4, Dor: $10.1049 / \mathrm{cp} .2009 .0112$.

[11] D. A. Garren, P. E. Pace and R. A. Romero, "Use of P3-coded transmission waveforms to generate synthetic aperture radar images," in Proc. 2014 IEEE Radar Conf., Cincinnati, OH, USA, 2014, pp. 0765-0768, DoI: 10.1109/RADAR.2014.6875692.

[12] E. Mozeson and N. Levanon, "Removing autocorrelation sidelobes by overlaying orthogonal coding on any train of identical pulses," IEEE Trans. Aerosp. Electron. Syst., vol. 39, no. 2, pp. 583-603, Apr. 2003, DoI: 10.1109/TAES.2003.1207268.

[13] R. Sivaswamy, "Digital and analog subcomplementary sequences for pulse compression," IEEE Trans. Aerosp. Electron. Syst., vol. AES14, no. 2, pp. 343-350, Mar. 1978, Dor: 10.1109/TAES.1978.308657.

[14] N. Levanon and E. Mozeson, Radar Signals. Hoboken, NJ, USA: Wiley, 2004.

[15] D. Chebanov and G. Lu, "Removing autocorrelation sidelobes of phase-coded waveforms," in Proc.IEEE Radar Conf., Washington, DC, USA, 2010, pp. 1428-1433, DoI: 10.1109/RADAR.2010.5494391.

[16] R. N. Ipanov, A. I. Baskakov, N. Olyunin and M.-H. Ka, "Radar Signals with ZACZ Based on Pairs of D-Code Sequences and Their Compression Algorithm," IEEE Signal Proc. Lett., vol. 25, no. 10, pp. 1560-1564, Oct. 2018, Dor: 10.1109/LSP.2018.2867734.

[17] R. N. Ipanov, "Pulsed Phase-Shift Keyed Signals with Zero Autocorrelation Zone," J. of Commun. Techn. and Electron., vol. 63, no. 8, pp. 895-901, Aug. 2018, Dor: 10.1134/S1064226918080077.

[18] R. N Ipanov, "Signals with Zero Autocorrelation Zone for the Synthesized Aperture Radar," IET El. Lett., vol. 55, no. 19, pp. 10631065, Sep. 2019, Dor: 10.1049/el.2019.1918.

[19] R. N. Ipanov, "The formation method of the ensemble variety of p-ary D-codes, RU Patent 2670 773, Oct. 25, 2018.

[20] V.P. Ipatov, Spread Spectrum and CDMA, Principles and Applications. Hoboken, NJ, USA: Wiley, 2005.

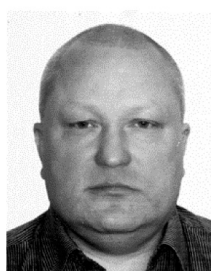

Roman N. Ipanov was born in Kishinev City, Moldova, USSR, in 1974. In 1999 he graduated the Military Space Academy named after A. F. Mozhaisky, Saint Petersburg (in the radio-electronic systems specialty). He received the $\mathrm{Ph} . \mathrm{D}$. in the radiolocation and radio navigation specialty at the Military Space Academy named after A. F. Mozhaisky in 2004 From 2004 to 2010, he worked as a lecturer and a senior lecturer in the department of radar technology at the Military Space Academy named after A. F.

Mozhaisky. From 2013 to 2010, he worked as a senior researcher in the Federal Government Institution of Scientific and Production Association in Special Equipment and Telecoms of the Ministry of Internal Affairs of the Russian Federation, Moscow. Currently, works as the Associate Professor at the Department of Radio Engineering Devices and Antenna Systems at the Moscow Power Engineering Institute, studying as Dr.Sci. candidate. He is the author of more than 50 scientific papers and three inventions. Research interests include the radar signals synthesis and digital processing. 